EPJ Web of Conferences 97,00001 (2015)

DOI: 10.1051/epjconf/20159700001

(C) Owned by the authors, published by EDP Sciences, 2015

\title{
Why hadronic resonances and particle unstable states are inter- esting?
}

\author{
J. Aichelin \\ ${ }^{1}$ SUBATECH, Laboratoire de Physique Subatomique et des Technologies Associées, \\ Université de Nantes - IN2P3/CNRS - Ecole des Mines de Nantes \\ 4 rue Alfred Kastler, F-44072 Nantes, Cedex 03, France
}

\begin{abstract}
We discuss the (few) achievements and the (many) open questions of the theoretical interpretation of the recent data on unstable particles in pp, pA and AA collisions at ultrarelativistic energies.
\end{abstract}

\section{Introduction}

Why we are here at this second resonance workshop which is by far larger than the first one in Austin two years ago? The reason is very simple. High precision data from the LHC experiments exist by now, we have the first results from the beam energy scan at RHIC and an increasing number of detailed data of the high energy RHIC runs. All this data force us to realize that the heavy ion reaction is more complex than the first blast wave fits and statistical model comparisons have suggested. Especially if we want to get a comprehensive understanding of the proton-proton (pp), proton- heavy ion (pA) and heavy ion - heavy ion (AA) data we have to realize that the simple picture that in central AA collisions a plasma of quarks and gluons (QGP) is created which can be described in hydrodynamical models, that in pA collision we can study cold nuclear matter effects, which means essential the hadronic rescattering, whereas pp collisions are elementary in the sense that two partons of the incomming protons collide is not compatible with the experimental findings. High multiplicity pp, pA and AA data show unexpected common features like ridges and a mass dependence of $v_{2}\left(p_{T}\right)$ which have been expected for AA collisions only. They hint to collective effects and an hydrodynamical behaviour, means strong interactions among the particles, even in pp and pA collisions.

In this situation the study of the most frequent hadrons, like pions, kaons and protons, does not help very much. Their multiplicity in pp, pA AA collisions is very well described in a statistical model [1], even if in central AA collisions at LHC there is a $10 \%$ deviation of the protons. For central AA collisions at LHC we see this comparison in fig.1. Thus their multiplicity tells as little about their production mechanism and their interaction they had before they arrive at the detector. The temperature of $156 \mathrm{MeV}$ is close to the hadronization temperature of lattice gauge calculations at which quarks become bound into hadrons. This suggests that the chemical composition of the hadrons gas is fixed at that temperature. However, at an energy density of around $0.5 \mathrm{GeV} / \mathrm{fm}^{3}$, which corresponds to this transition temperature, the hadrons are densely packed and will interact still on the way to the detector. Fig.2, left; shows the hadronisation temperature $T_{c h}$, which determines the chemical composition of the hadron gas, as well as the kinetic temperature $T_{k i n}$ at which the hadronic

This is an Open Access article distributed under the terms of the Creative Commons Attribution License 4.0, which permits unrestricted use, distribution, and reproduction in any medium, provided the original work is properly cited. 


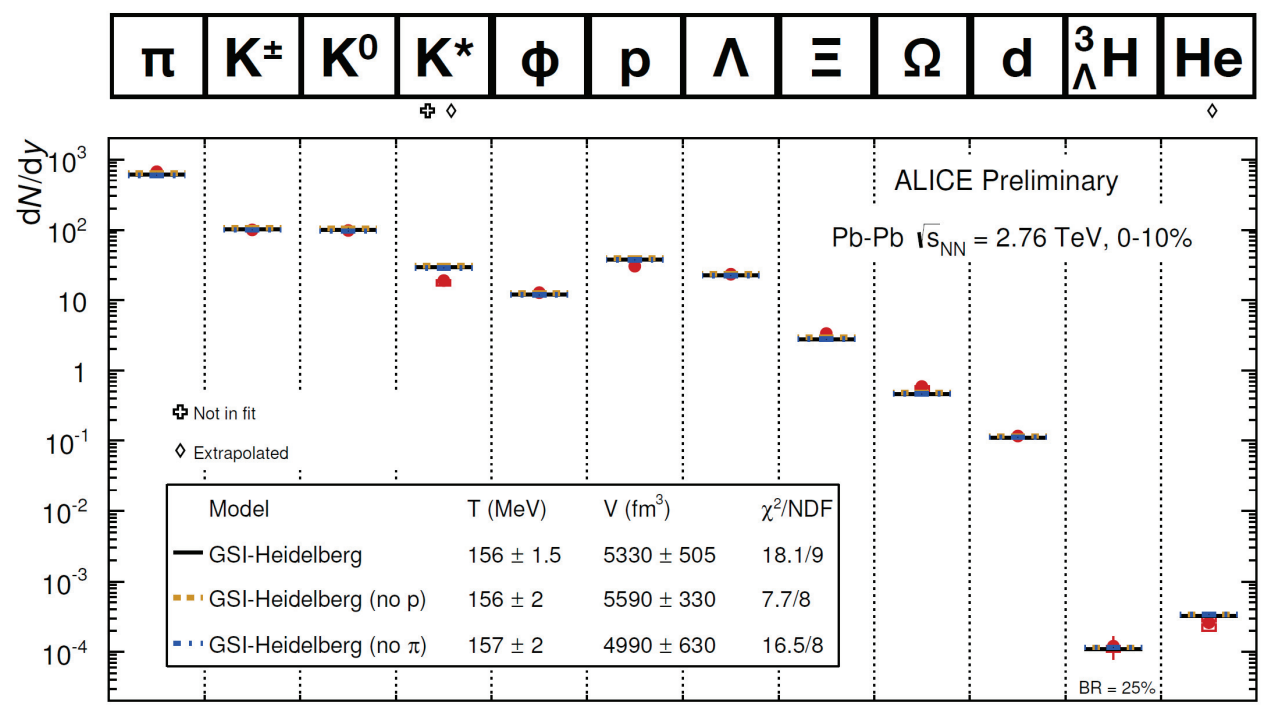

Figure 1. Multiplicities of selected hadrons in central $(0-10 \%) \mathrm{PbPb}$ collisions at $2.76 \mathrm{AGeV}$ as compared with the statistical model predicitions [1].

interactions cease [2]. We see that the difference between both becomes larger with increasing energy, leaving more room for hadronic rescattering. At the same time the collective expansion velocity, Fig. 2 right, increases and makes the expansion faster.
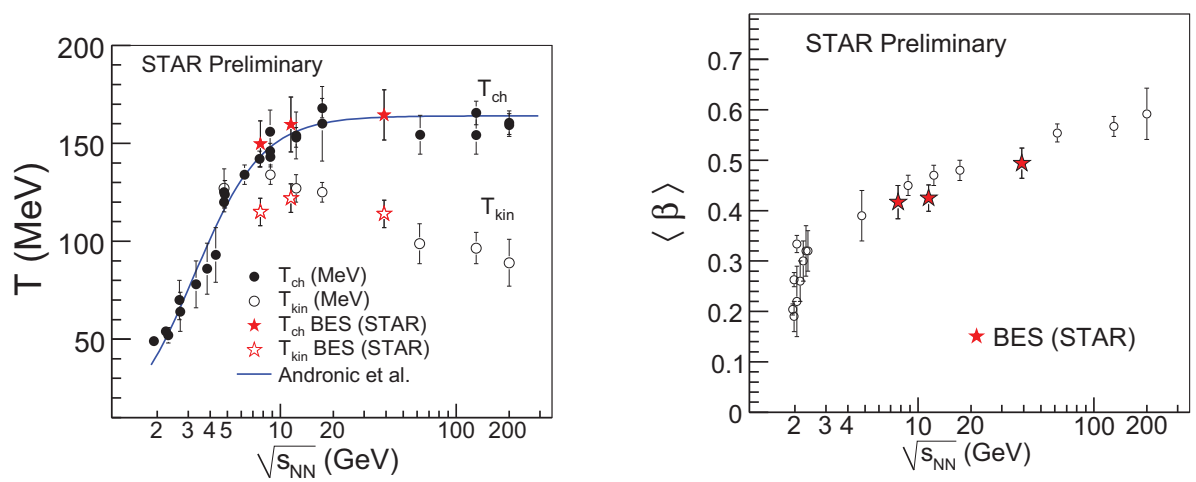

Figure 2. Left: Chemical freeze out temperature, $T_{c h}$, and kinetic freeze out temperature, $T_{k i n}$, as a function of $\sqrt{s}$. Right: average radial velocity as a function of $\sqrt{s}[2]$.

The ultimate challenge of the ultrarelativistic heavy ion physics is to understand the formation, expansion and hadronization of the QGP. This signal is distorted by the hadronic interactions which take place between $T_{c h}$ and $T_{k i n}$ and which change considerably the momentum distribution of the hadrons. Therefore we have to understand this region. In addition, this is the region of high density 
hadron physics which is in itself of great interest, theoretically as well as experimentally. Presently there are two transport approaches available which can deal with the physics between $T_{c h}$ and $T_{k i n}$. The ultrarelativistic quantum molecular dynamics (UrQMD) approach [4] and the parton hadron string model (PHSD) [5]. UrQMD calculations, Fig. 3, show that in reality also the chemical composition changes between $T_{c h}$ and $T_{k i n}$. For $\mathrm{PbPb}$ at $\sqrt{s}=2.76 \mathrm{ATeV}$ we display on the left hand side the $p_{T}$ integrated modification factor (the ratio of finally observed particles to the one produced at $T_{c h}$ ) of different hadrons as a function of the centrality and on the right hand side the modification factor as a function of $p_{T}$ for the most central events $(0-10 \%)$. We see modifications up to $25 \%$ which concern mostly the low $p_{T}$ hadrons for which the phase space occupation is largest.
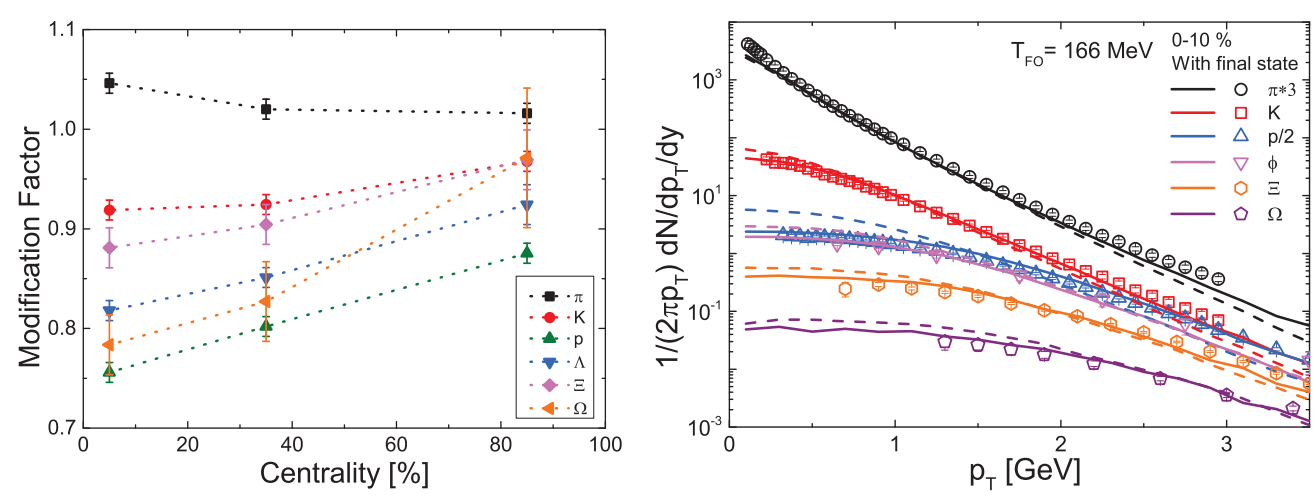

Figure 3. Modification of the $p_{T}$ integrated yield as a function of centrality for different hadrons (left) and as a function of $p_{T}$ for central events $(0-10 \%)$ during the hadronic rescattering phase, for $\mathrm{PbPb}$ at $\sqrt{s}=2.76 \mathrm{ATeV}$ [3].

Resonances may elucidate this region in several ways:

- Their measured multiplicity as compared to the expected multiplicity in statistical model calculations provides information about the interaction time and the implied cross section because experimentally only those resonances can be reconstructed whose decay products did not have collisions with surrounding hadrons. Exploiting the different life times of the different resonances we may reconstruct, with help of transport approaches, the whole time evolution of the expansion of the hadron gas.

- Resonances which decay into photons or dileptons measure the total time of the hadronic expansion because any produced resonance contributes, even if it is later reabsorbed. Thus, the longer the hadronic gas interacts the more photons or dileptons are produced. Together with the elliptic flow signal (which is finite for hadronically produced photons) they may provide information about the contribution from the plasma and hence about its space time evolution.

- Neither heavy quarks nor heavy mesons come to an equilibrium with their environment. Their spectra offers therefore informations on their interaction with the plasma constituents and the hadron gas, respectively. The multiplicity ratio of excited states of heavy mesons, like $\psi(2 S)$ or $Y(2 S)$ and $Y(3 S)$, and the ground states provides in addition information on the hadronic rescattering. In view of their large binding energies this ratio is especially sensitive to violent collisions. 


\section{Hadronic Decays}

Hadronicly decaying resonances have been measured at RHIC and LHC. The RHIC results from the STAR collaboration [6], covering very different life times of resonances $\left(\phi=44 \mathrm{fm} / \mathrm{c}, \Lambda^{*}=\right.$ $13 \mathrm{fm} / \mathrm{c}, \Sigma=5.7 \mathrm{fm} / \mathrm{c}, K^{*}=4 \mathrm{fm} / \mathrm{c}$ ), are shown in Fig. 4 and are compared with prediction from the thermal model [7] as well as from UrQMD calculations [8, 9]. Assuming only resonance decay

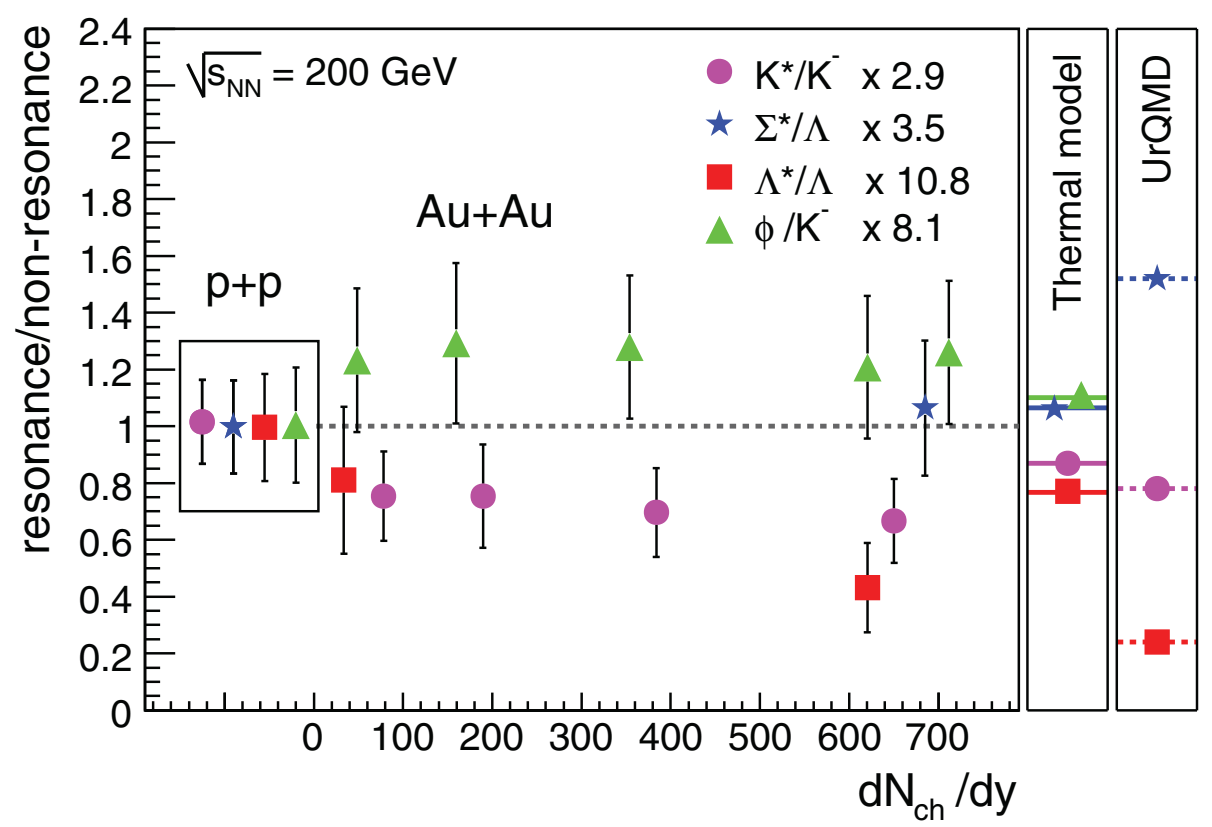

Figure 4. Resonance production at RHIC. We compare the STAR result [6] with thermal predictions [7] and with those from UrQMD calculations $[8,9]$.

one would expect that the displayed ratio decreases for short living resonances more strongly with centrality than for long living ones. The experiment supports this expectation only partially. We see a strong decrease of the $\Lambda^{*} / \Lambda$ ratio and an almost constant $\phi / K^{-}$ratio but the $K^{*} / K$ ratio is centrality independent and for the $\Sigma^{*} / \Lambda$ ratio we find in central heavy ion collisions the same value as in pp collisions. Evidentially regeneration of resonances cannot be neglected and because most of these regeneration cross sections are little known, if at all, this poses problems for a theoretical interpretation of the data as the UrQMD calculations show for central collisions. They show the right trend but differ in details. Statistical models should overpredict the yield because they neglect the rescattering of the decay products. This we see for the $\Lambda^{*} / \Lambda$ and the $K^{*} / K$ ratio but not for the $\Sigma^{*} / \Lambda$ ratio. The resonance suppression is at most $60 \%$ but usually considerably smaller. If one wants to draw conclusions about the hadronic interaction region with help of resonances high accuracy measurements with a little systematic error are necessary. At LHC for most of the resonances spectra have not been published yet. Form Fig. 1 we see that $K^{*}$ are about $40 \%$ less frequently produced than expected from the statistical model. Hence the suppression is even a bit larger than at RHIC. A detailed comparison with theory is not at hand yet but transport theories show as well a strong suppression of 
the resonances due to hadronic rescattering. In Fig. 5 we display the $p_{T}$ distribution of EPOS [10] of selected resonances immediately after hadronization as well as that of resonances which can be reconstructed finally by the invariant mass method, means of those whose decay products do not have rescattered. The hadronic rescattering in the EPOS approach is identical to the UrQMD model. We see that in the most central events $(5-10 \%)$ up to $80 \%$ of the resonances cannot be reconstructed. It remains to be seen whether these findings coincide with experiments.
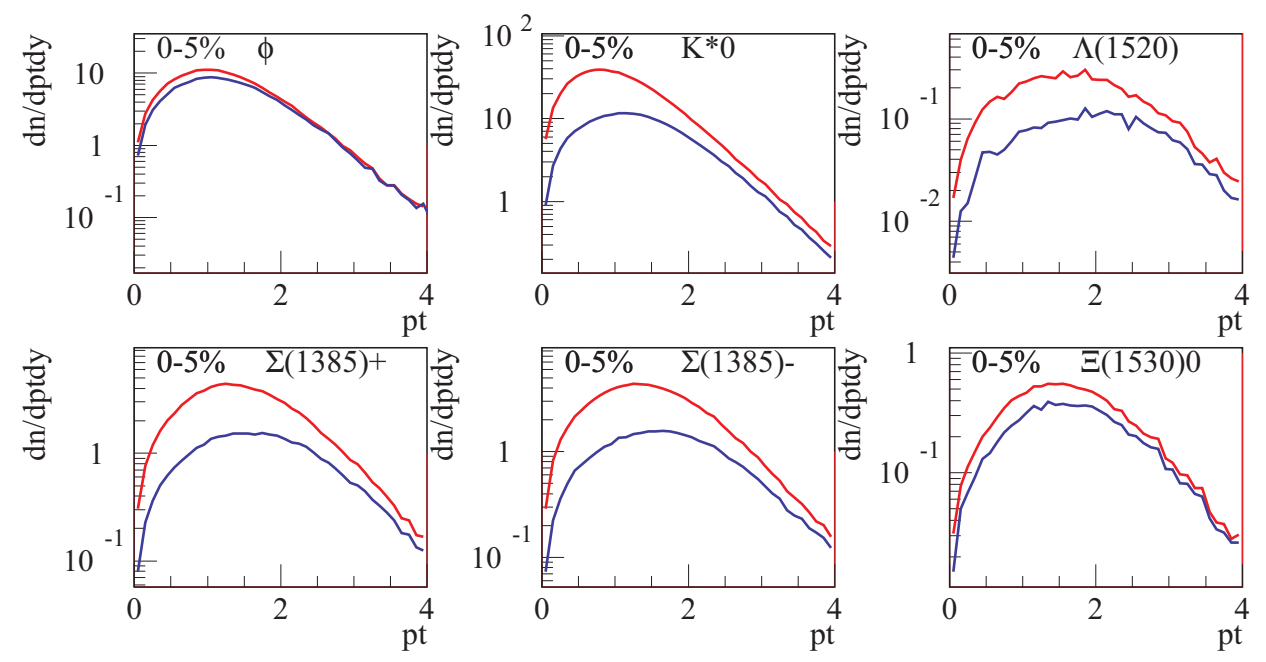

Figure 5. $p_{T}$ distribution of different resonances in the EPOS model [10]. For several resonances we display the $p_{T}$ distribution immediately after hadronzation (red line) of the plasma and that of those resonances which can be finally reconstructed by the invariant mass method.

At the moment all the theoretical models use measured free cross sections or parameterizations if measured cross sections are not available. Recently it has been shown that at finite temperature cross sections will be modified [11]. To take this into account provides a new task for the transport approaches.

\section{Electromagnetic Probes}

Photons and dileptons are a very valuable probe to study the time evolution of the expanding system because they do not get reabsorbed. They are emitted from the initial collision of the incoming nuclei, while the plasma is formed and expanding and finally also during the hadronic phase. Initially there was the idea to measure the direct photons, i.e. those emitted from the plasma, by subtracting from the inclusive spectra the contribution of the measured yield of hadronic electromagnetic sources like $\omega, \phi$ and $\eta^{\prime}$ to the photon spectrum. It came as a surprise when the PHENIX collaboation measured that $v_{2}$ of direct photons is almost as large as that of hadrons. If photons from the plasma dominate the direct photon yield one would expect a smaller value because the $v_{2}$ develops during the plasma expansion and early emitted photons do not have an elliptic flow. In the mean time this puzzle seems to be solved [13, 14]. They showed that the direct photons, as defined above, are not only those emitted 
from the plasma. In addition, two hadronic sources contribute which have been neglected before: the bremsstrahlung between charged hadrons and photon emission from intermediate hadrons which are subsequently reabsorbed and do not appear in the observed hadron spectrum. If one includes these additional sources a good agreement with the experimental data can be found, for the direct photon yield as well as for their $v_{2}$, as can be seen in Fig. 6 . There we display on the left hand side the direct photon yield [12] in comparison with the simulations $[13,14]$. The simulations are subdivided into the different contributions. On the right hand side one sees the elliptic flow of direct photons. Because hadronic sources are important we expect, as seen, a finite elliptic flow. A similar good
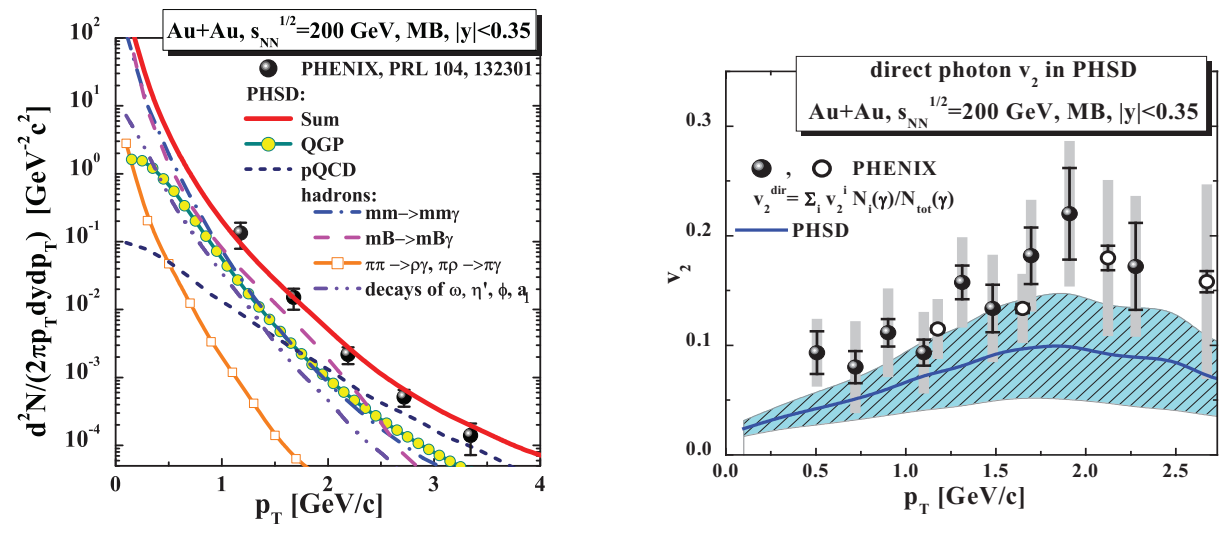

Figure 6. Left: Direct photon $p_{T}$-spectrum from the PHSD approach in comparison to the PHENIX data [12] for midrapidity minimal bias Au+Au collisions at $\sqrt{s}=200 \mathrm{GeV}$. Right: Direct photon elliptic flow coefficient $v_{2}\left(p_{T}\right)$ from the PHSD approach in comparison to the PHENIX data [12] for midrapidity minimal bias $\mathrm{Au}+\mathrm{Au}$ collisions at $\sqrt{s}=200 \mathrm{GeV}$. The figures are taken from [13].

agreement between experiment and theory, even for two independent transport approaches, has been found for dileptons. In Fig. 7 we display the centrality dependence of the midrapidity dilepton yield (left) and its ratio to the 'cocktail' for 4 centrality bins, $0-10 \%, 10-40 \%, 40-80 \%, 0-80 \%$, for central $\mathrm{Au}+\mathrm{Au}$ collisions at $\sqrt{s}=200 \mathrm{GeV}$. The agreement between the calculations and the data shows that below $M_{e e}=0.8 \mathrm{GeV}$ medium effects become important which are not contained in the cocktail. The reproduction of the resonance peaks in the dilepton spectrum and the reproduction of the photon yield show that the hadronic expansion phase in which each collision of a charged hadron adds to the photon yield and each resonance which possibly decays into dileptons contributes to the dilepton yield seems to be under control. Photons and dilepton calculations are presently closer to experiment than all other probes discussed here.

\section{Excited States of Heavy Flavour Mesons}

The physics of excites states of charm and bottom mesons is the newest branch of the physics of unstable particles in ultrarelativistic heavy ion physics. Both, at RHIC and at LHC, the results are so surprising that a theoretical understanding is far from being achieved. In free space heavy mesons are deeply bound (1100 MeV, $535 \mathrm{MeV}$ and $203 \mathrm{MeV}$ for Y(1S), Y(2S) and Y(3S), respectively and 642 $\mathrm{MeV}$ and $53 \mathrm{MeV}$ for $\mathrm{J} / \psi$ and $\psi(2 S)$, respectively) and their radii are small $\mathrm{Y}(1 \mathrm{~S}) \approx 0.2 \mathrm{fm}, \mathrm{Y}(2 \mathrm{~S}) \approx .5$ $\mathrm{fm}, \mathrm{Y}(3 \mathrm{~S}) \approx .75 \mathrm{fm}, \mathrm{J} / \psi \approx .4 \mathrm{fm}$ and $\psi(2 S) \approx .85 \mathrm{fm}$. The ALICE collaboation has measured the $J / \psi$ 

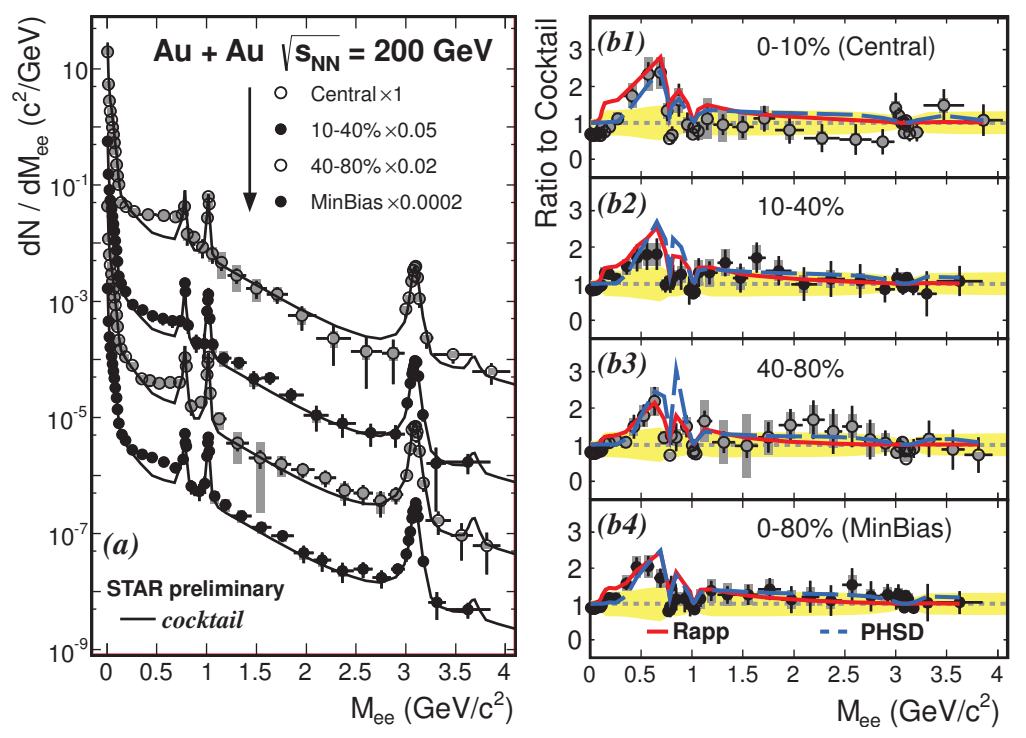

Figure 7. Centrality dependence of the midrapidity dilepton yields (left) and its ratios (right) to the 'cocktail' for $0-10 \%, 10-40 \%, 40-80 \%, 0-80 \%$ central Au+Au collisions at $\sqrt{s}=200 \mathrm{GeV}$ : a comparison of STAR data with theoretical predictions from the PHSD ('PHSD' - dashed lines) and the expanding fireball model ('Rapp' - solid lines). The figure is taken from Ref.[15] .

and $\psi(2 S)$ yield in pA collisions at $\sqrt{s}=5.02 \mathrm{AGeV}$ in forward and backward rapidity, see Fig.8. The nuclear modification factor $R_{p P b}$ for $\psi(2 S)$ is unexpectedly low and questions our understanding of the formation of charmonium states which consists in the assumption that in a hard collision a $c \bar{c}$ pair is formed locally and expands during the formation time $\tau_{\mathrm{f}}$ before a $J / \psi$ or a $\psi(2 S)$ state can be identified [18]. One may naively assume that the small $R_{p P b}$ comes from the cold nuclear matter environment where the less bound $\psi(2 S)$ can be easily destroyed. This requires, however, that the formation time of the charmonium, $\tau_{\mathrm{f}}$, is shorter than the time the charmonium needs to traverses the nucleus, $\tau_{\mathrm{c}}$. One can evaluate the average proper time $\tau_{\mathrm{c}}$ spent in the nucleus as $\tau_{\mathrm{c}}=\langle L\rangle /\left(\beta_{\mathrm{z}} \gamma\right)$ [19], where $\langle L\rangle$ is the average length of nuclear matter crossed by the pair. For $c \bar{c}$ pairs in the charmonium mass range emitted at $p_{\mathrm{T}}=0$ in the forward acceptance, one gets $\tau_{\mathrm{c}} \sim 10^{-4} \mathrm{fm} / c$, while the corresponding value at backward rapidity is $\sim 7 \cdot 10^{-2} \mathrm{fm} / c$. Estimates for the formation time $\tau_{\mathrm{f}}$ range between 0.05 and $0.15 \mathrm{fm} / c$ [18]. Hence we do not expect differences between $J / \psi$ and $\psi(2 S)$ in forward direction and even in backward direction we find at most $\tau_{\mathrm{f}} \approx \tau_{\mathrm{c}}$. Therefore, according to our present understanding of the charmonia production the small $R_{p P b}$ value cannot come from hadronic rescattering. Thus we are left with the question whether our model for the production of charmonia is not correct or whether other origins, like medium modifications of the production processes, have to be considered. Fig. 8 shows as well that shadowing and energy loss cannot reconcile the data with this theoretical approach. For more details we refer to [16].

Another challenge for theory has been presented by the CMS collaboration[22]. They showed, Fig. 9, left, that even for the strongly bound $\mathrm{Y}(2 \mathrm{~S})$ state we see already in pA collisions a suppression 


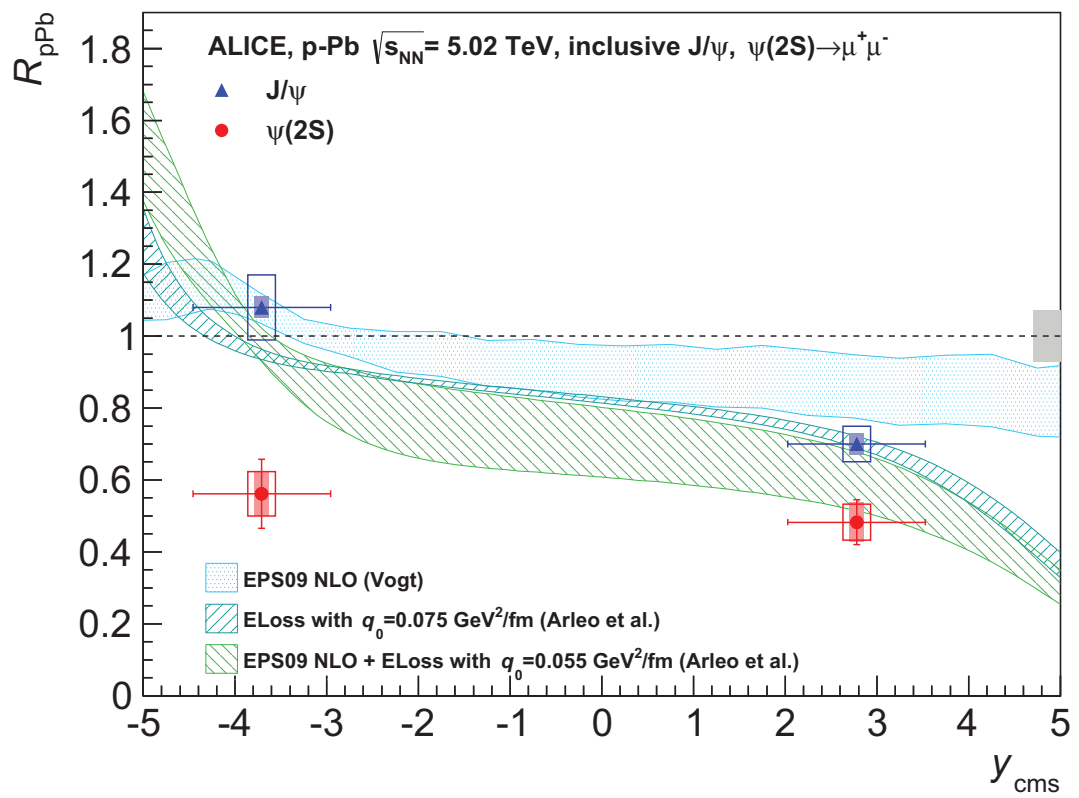

Figure 8. The nuclear modification factor for $\psi(2 \mathrm{~S})$, compared to the corresponding quantity for $\mathrm{J} / \psi[16]$. The horizontal bars correspond to the width of the rapidity regions under study. The vertical error bars correspond to statistical uncertainties.
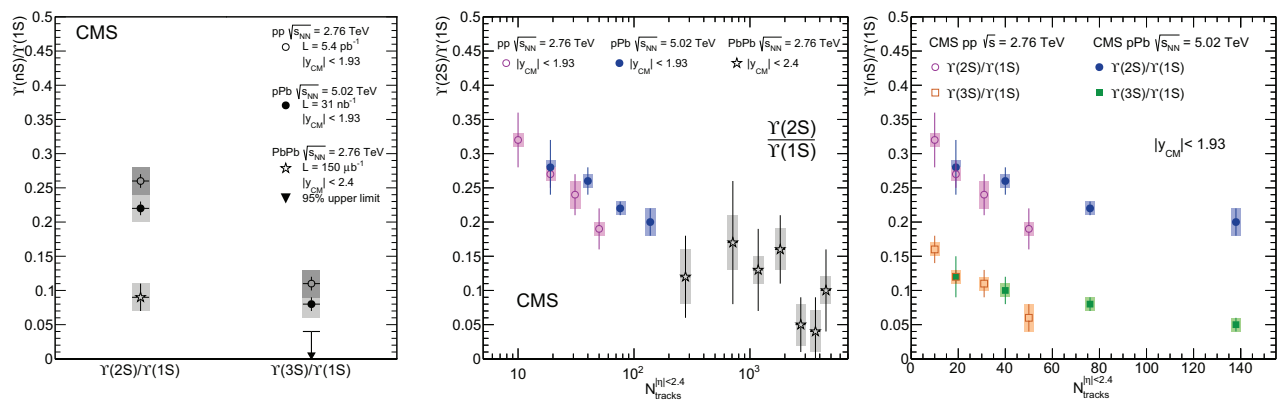

Figure 9. Left: The ratios $\mathrm{Y}(2 \mathrm{~S}) / \mathrm{Y}(1 \mathrm{~S})$ and $\mathrm{Y}(3 \mathrm{~S}) / \mathrm{Y}(1 \mathrm{~S})$ in minimum bias $\mathrm{pp}$, pA and AA collisions. We see a suppression of the excited states in $\mathrm{pA}$ ( cold nuclear matter effect) and an even stronger suppression in AA collisions (hot matter effect). Middle: The $\mathrm{Y}(2 \mathrm{~S}) / \mathrm{Y}(1 \mathrm{~S})$ ratio as a function of the track multiplicity in pp, pA and AA collisions. Right: The $\mathrm{Y}(3 \mathrm{~S}) / \mathrm{Y}(1 \mathrm{~S})$ ratio as a function of the track multiplicity in pp and $\mathrm{pA}$ collisions. All figures are from [22].

as compared to pp collisions and an even larger suppression in AA. What is even more astonishing is the dependence of the ratio of $\mathrm{Y}(2 \mathrm{~S}) / \mathrm{Y}(1 \mathrm{~S})$, middle, and of $\mathrm{Y}(3 \mathrm{~S}) / \mathrm{Y}(1 \mathrm{~S})$, right, on the track multiplicity. This dependence is surprisingly similar for $\mathrm{pp}, \mathrm{pA}$ and AA collisions despite of the very different geometries and kinematic conditions. For our understanding it would be interesting to know whether the production depends on the number of associated particles or whether already in pp colli- 
sions further collisions between the produced particles can suppress the yield. Up to now there is no theoretical explanation.

I hope that I have demonstrated, using some examples, that the physics of unstable particles presents a lot of challenges, experimentally as well as theoretically, but most of all it is a very valuable tool to explore these reactions in detail. I hope that this workshop contributes to our understanding.

\section{References}

[1] M. Floris Invited talk at the workshop "QCD Hadronization and the Statistical Model" at the ECT* Trento, oct 6-10, 2014 http://www.ectstar.eu/node/790

[2] L. Kumar [STAR Collaboration], J. Phys. G 38 (2011) 124145 [arXiv:1106.6071 [nucl-ex]].

[3] F. Becattini, M. Bleicher, T. Kollegger, T. Schuster, J. Steinheimer and R. Stock, Phys. Rev. Lett. 111, 082302 (2013) and proceedings of this conference

[4] S. A. Bass, M. Belkacem, M. Bleicher, M. Brandstetter, L. Bravina, C. Ernst, L. Gerland and M. Hofmann et al., Prog. Part. Nucl. Phys. 41 (1998) 255 [Prog. Part. Nucl. Phys. 41 (1998) 225] [nucl-th/9803035].

[5] W. Cassing, E.L. Bratkovskaya, Phys. Rept. 308 (1999) 65.

[6] B. I. Abelev et al. [STAR Collaboration], Phys. Rev. Lett. 97 (2006) 132301 [nucl-ex/0604019].

[7] P. Braun-Munzinger, D. Magestro, K. Redlich and J. Stachel, Phys. Lett. B 518 (2001) 41 [hep$\mathrm{ph} / 0105229]$.

[8] M. Bleicher and J. Aichelin, Phys. Lett. B 530 (2002) 81 [hep-ph/0201123].

[9] M. Bleicher and H. Stoecker, J. Phys. G 30 (2004) S111 [hep-ph/0312278].

[10] K. Werner, B. Guiot, I. Karpenko and T. Pierog, Phys. Rev. C 89 (2014) 064903 [arXiv:1312.1233 [nucl-th]] and K. Werner, private communication

[11] D. Cabrera, L. Tolós, J. Aichelin and E. Bratkovskaya, Phys. Rev. C 90 (2014) 5, 055207 [arXiv:1406.2570 [hep-ph]].

[12] A. Adare et al. [PHENIX Collaboration], Phys. Rev. Lett. 109 (2012) 122302 [arXiv:1105.4126 [nucl-ex]].

[13] E. L. Bratkovskaya, arXiv:1408.3674 [hep-ph].

[14] O. Linnyk, W. Cassing and E. L. Bratkovskaya, Phys. Rev. C 89 (2014) 3, 034908 [arXiv:1311.0279 [nucl-th]].

[15] Y. Guo [STAR Collaboration], J. Phys. Conf. Ser. 535 (2014) 012006 [arXiv:1407.6788 [hepex]].

[16] B. B. Abelev et al. [ALICE Collaboration], arXiv:1405.3796 [nucl-ex].

[17] B. Abelev et al. (ALICE Collaboration), JHEP 1402 (2014) 073.

[18] F. Arleo, P. B. Gossiaux, T. Gousset and J. Aichelin, Phys. Rev. C 61 (2000) 054906 [hep$\mathrm{ph} / 9907286]$.

[19] D. McGlinchey, A.D. Frawley and R. Vogt, Phys. Rev. C87 (2013) 054910.

[20] J.L. Albacete et al., Int. J. Mod. Phys. E22 (2013) 1330007 and priv. comm.

[21] F. Arleo and S. Peigné, JHEP 1303 (2013) 122.

[22] S. Chatrchyan et al. [CMS Collaboration], JHEP 1404 (2014) 103 [arXiv:1312.6300 [nucl-ex]]. 
